\title{
Polarization by light reflection at metallic surfaces observed in the shape of the Balmer-\# line of low density plasmas
}

Cite as: Phys. Plasmas 26, 073513 (2019); https://doi.org/10.1063/1.5088931

Submitted: 15 January 2019 . Accepted: 16 June 2019 . Published Online: 29 July 2019

Sven Dickheuer (D), Oleksandr Marchuk (D), Yuri Krasikov (D), Philippe Mertens (D), Christian Brandt (D), Stephan Ertmer (D), Petra Börner (D), Detlev Reiter (D), Beatrix Göths, Kalle von Bovert, and Arkadi Kreter (i)
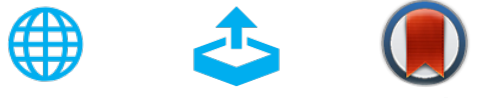

\section{ARTICLES YOU MAY BE INTERESTED IN}

On the generalized formulation of Debye shielding in plasmas

Physics of Plasmas 26, 050701 (2019); https://doi.org/10.1063/1.5091949

Studies on virtual electrode and ion sheath characteristics in a cylindrical inertial electrostatic confinement fusion device

Physics of Plasmas 26, 073514 (2019); https://doi.org/10.1063/1.5107471

Ion-acoustic waves in dense magneto-rotating quantum plasma

Physics of Plasmas 26, 072119 (2019); https://doi.org/10.1063/1.5082868

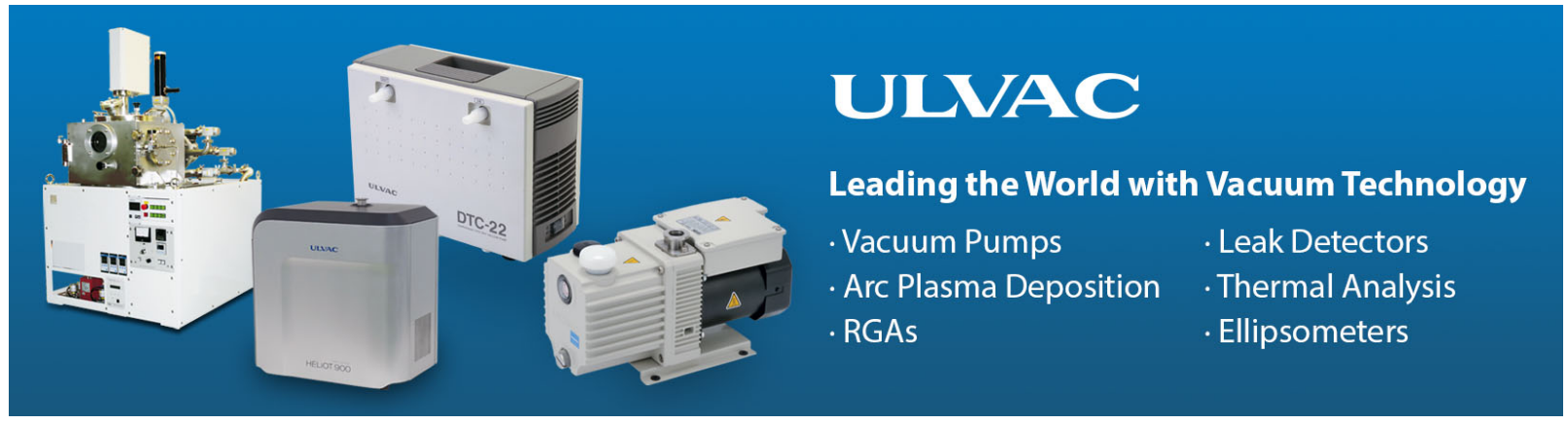




\title{
Polarization by light reflection at metallic surfaces observed in the shape of the Balmer- $\alpha$ line of low density plasmas
}

Cite as: Phys. Plasmas 26, 073513 (2019); doi: 10.1063/1.5088931

Submitted: 15 January 2019 - Accepted: 16 June 2019 .

Published Online: 29 July 2019
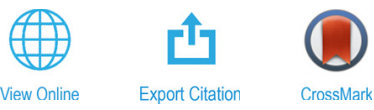

Sven Dickheuer, (D) Oleksandr Marchuk, ${ }^{a)}$ (D) Yuri Krasikov, (iD Philippe Mertens, (iD Christian Brandt, ${ }^{\text {b) }}$ (iD Stephan Ertmer, (iD Petra Börner, (D) Detlev Reiter, (D) Beatrix Göths, Kalle von Bovert, and Arkadi Kreter (D)

\author{
AFFILIATIONS \\ Jülich $\mathrm{GmbH}, 52425$ Jülich, Germany

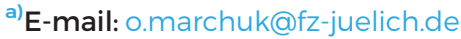

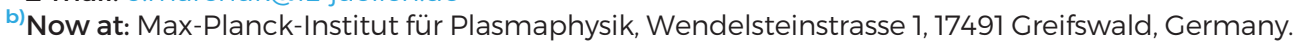

Institut für Energie- und Klimaforschung - Plasmaphysik, IEK-4, Partner of the Triliteral Euregio Cluster (TEC), Forschungszentrum

\begin{abstract}
The degree of linear polarization of light reflected at metallic surfaces is obtained from the shape of the Balmer- $\alpha$ line measured in a low density plasma. The measurements of the polarization properties of metallic surfaces utilize only the high-resolution emission spectra induced by fast reflected $\mathrm{H}$ atoms $(\approx 100 \mathrm{eV})$ in ArH plasmas. The measurements are performed at two different lines-of-sight to the target surfaces of $\mathrm{Mo}$ and $\mathrm{Cu}$. Only at the observation angle close to the Brewster one, a significant drop of the measured red-shifted signal is detected for the Mo target: the red-shifted emission reduces by $\approx 50 \%$ depending on whether the transverse $(\mathrm{S})$ or the parallel $(\mathrm{P})$ polarization component of the reflected light is selected. In contrast to this, a very weak change of emission is observed for the Cu surface for the same angle. The spectra measured in front of the Mo target were modeled utilizing the energy and angular distribution of reflected atoms and excitation of the cross section of Ar-H collision combined in a Doppler-shifted emission model. A good agreement with the theoretical calculations is found for polarization components (except for the weak P polarization at $70^{\circ}$ ). Finally, the undercosine distribution of the reflected atoms with $b \approx 0.2$ ( $b$ is the power of the cosine distribution) shows the best agreement with the spectra measured at both lines-of-sight.
\end{abstract}

Published under license by AIP Publishing. https://doi.org/10.1063/1.5088931

\section{INTRODUCTION}

Polarization of light reflected at metallic surfaces represents one of the basic phenomena of light-matter interaction being extensively used in the fundamental and industrial research such as physics of solids, physics of thin films, or laser physics. Specifically, the sensitivity of polarization to the surface morphology, e.g., the transition from the specular to the diffusive type of reflectance, makes polarization indispensable in many areas of optics.

However, accurate spectroscopic measurements of the polarized reflectance of metallic surfaces during plasma operation do not exist: External light sources such as lasers have to be installed. Measurements of polarization in astrophysical and laboratory plasmas refer primarily to phenomena of anisotropic excitation of spectral lines induced by particle collisions, ${ }^{3}$ by the impact of electromagnetic fields, ${ }^{4,5}$ or by combinations of any of them. ${ }^{6,7}$ The measurements of polarization of plasma facing components and its dynamics would be indispensable in terms of numerous applications of plasma treatment of the surface or due to the sensitivity of plasma diagnostics to the polarization properties of optical components. ${ }^{8,9}$

It was shown recently that in low density (the gas pressure is about $0.05 \mathrm{~Pa}$, and the plasma density is $10^{11} \mathrm{~cm}^{-3}$ ) and low temperature (the electron temperature is $3-10 \mathrm{eV}$ ) plasma discharges, the excitation of fast hydrogen atoms in the energy range of $100-300 \mathrm{eV}$ in collisions with Ar is much more efficient compared to excitation by $\mathrm{Kr}$ or by other noble gases or by electron collisions. ${ }^{10}$ Moreover, the Doppler effect in the front of the surface could replace the timeresolved measurements of reflectance in the laboratory to a certain extent. ${ }^{11-13}$ The measurements of the optical properties of metals at high temperatures or monitoring the degradation of optical properties are examples of new applications. The DSRM (Doppler-Shifted Reflectance Measurements) diagnostic relies crucially on the fact that in such ArH plasmas, the hydrogen atoms reflected at metallic surfaces with the kinetic energy of 100-300 eV emit the photons only after they pass the Child-Langmuir sheath. If the emission of reflected atoms is 
isotropic, the observed emission is a superposition of two noninteracting signals: (i) the blue-shifted, or the reference signal, is nonpolarized, ${ }^{14}$ whereas (ii) the red-shifted signal, being a result of light reflection at the surface, must contain the necessary information on the degree of polarization by reflection. The change of the signal due to the polarization properties of a W surface was already demonstrated in Ref. 13; however, the values of polarization could not be extracted since at large observation angles, the direct and the reflected emission intervals overlap significantly. Indeed, the direct emission appears at the spectral interval according to the Doppler effect at $\left[\lambda_{0}\left(1-v_{0} / c\right)\right.$, $\left.\lambda_{0}\left(1+v_{0} / c \sin \left(\theta_{0}\right)\right)\right]$ and the reflected signal at $\left[\lambda_{0}\left(1-v_{0} /\right.\right.$ $\left.\left.c \sin \left(\theta_{0}\right)\right), \lambda_{0}\left(1+v_{0} / c\right)\right]$. Here, $\lambda_{0}$ is the wavelength of the transition, $c$ is the speed of light in vacuum, $v_{0}$ is the maximal velocity of reflected atoms, and $\theta_{0}$ is the observation angle between the line-of-sight and the surface normal. Thus, the modeling of emission spectra is required to derive the values of polarization for large observation angles.

A similar type of modeling was already performed in high pressure $(\approx 100 \mathrm{~Pa})$ pure $\mathrm{H}$ or $\mathrm{D}$ gas discharges; ${ }^{15,16}$ however, the optical properties of the surfaces were not considered there. Instead, the relative concentrations and the corresponding reactions of $\mathrm{H}^{+}, \mathrm{H}_{2}^{+}$, or $\mathrm{H}_{3}^{+}$ ions played the decisive role, as the red-shifted signal is dominated by the fast atoms approaching the cathode. One open question is the type of angular distribution of the fast approaching atoms [Fig. 12(b), Ref. 15]. These atoms are created as a result of charge-exchange between the accelerated ions in the sheath and the background gas. ${ }^{16}$ Until now, it is not clear why such atoms demonstrated instead "beamlike" diffusive angular distributions $(b=1)$ approaching the surface.

In the low density plasma discharges, only the reflected atoms could be detected, which simplifies the description considerably. The experimental data on the angular distribution of the reflected atoms in the low energy range of incident ions represent the special interest on its own. The routine measurements using ion beams are only performed at energies of a few kilo-electron-volts and the experimental data for the angular distribution of $\mathrm{H}$ or $\mathrm{D}$ atoms in the energy range of $50-200 \mathrm{eV}$ are hardly available. ${ }^{17}$ This low energy range below $1 \mathrm{keV}$ is of special interest, for instance, in fusion plasmas as the $\mathrm{H}^{+}$ ions, accelerated in the plasma sheath at the floating potential to the values of $3-5 T_{e}{ }^{18}$ with electron temperature $\mathrm{T}_{e}=10-50 \mathrm{eV}$ in the scrape-off layer, reenter the plasma predominantly as fast atoms. ${ }^{17}$ In this energy range one has to rely on the data from codes such as SRIM/TRIM. ${ }^{19,20}$ Benchmarked against experimental data in the high energy range, it is difficult to conclude on their reliability in the low energy range as rather controversial data are reported. The cosine distribution for reflected particles is established in the high energy limit of incident ions. ${ }^{17}$ The modeling of emission spectra of fast atoms provides not only the degree of polarization by light reflection at Balmer lines for specific metallic surfaces but also the angular distribution of reflected atoms in the energy range of $100 \mathrm{eV}$. This is the major aim of this paper.

\section{MEASUREMENTS OF THE BALMER- $\alpha$ LINE USING LINEAR POLARIZERS}

The measurements presented in this paper have been performed at the linear plasma device PSI-2. ${ }^{21}$ The top view of the measurements is shown schematically in Fig. 1. The plasma is generated at the hollow cathode (HC) and is driven toward the $z$ axis of the vacuum chamber

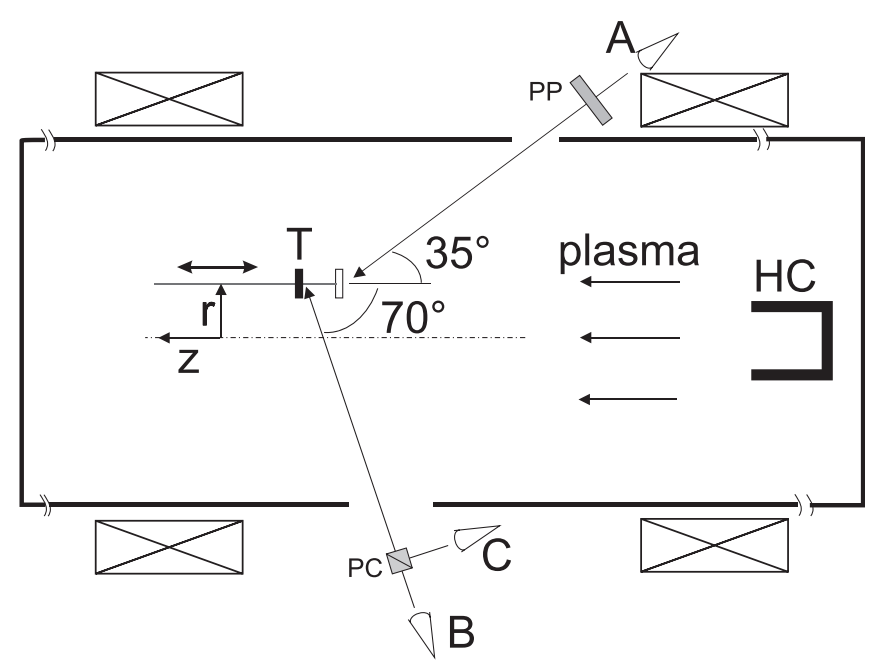

FIG. 1. Top view of the experimental setup at PSI-2 in the equatorial plane. The plasma is generated at the hollow cathode $(H C)$. The optical measurements are performed at the target $(T)$ positioned in one of the maxima of the hollow profile with $r=2.5 \mathrm{~cm}$. The axial manipulator carrying the target is moved along the $z$ axis to be in the line-of-sight of $35^{\circ}$ or $70^{\circ}$. For the $35^{\circ}$ observation, the polarization of emission was detected using the polarization plate $(P P)$ using the front optics $(A)$. For the $70^{\circ}$ observation, the polarization of emission was detected using the polarization cube $P C$ using the front optics $(B)$ and $(C)$.

by a pressure gradient. The magnetic coils generate the magnetic field of $\approx 0.1 \mathrm{~T}$. The operating plasma pressure used in the experiment is $0.01-0.1 \mathrm{~Pa}$. The plasma parameters are measured using the Langmuir probe at the distance of $1 \mathrm{~m}$ from the hollow cathode. The plasma profiles for electron density and temperature are exemplified in Fig. 2. Thus, in our measurements in ArH plasma, the electron temperature is in the range of $4-5 \mathrm{eV}$ and the electron density is about $4 \times 10^{11} \mathrm{~cm}^{-3}$.

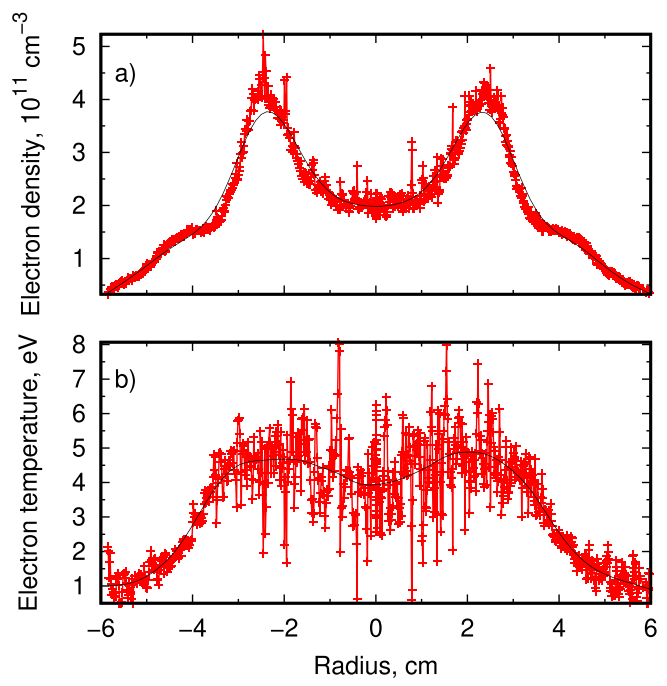

FIG. 2. Profiles of electron density (a) and electron temperature (b) measured using the Langmuir probe. The red line with points is the measurements, and the thin black line is the spline approximation. 
The spectra were measured at the lines-of-sight with the angles of $35^{\circ}$ and $70^{\circ}$ relative to the surface normal of the target $\mathrm{T}$ using the high resolution spectrometer. ${ }^{10}$ The choice of angles is determined by the access to the target surface and by the Brewster angle for Mo $\left(\approx 80^{\circ}\right)$ and $\mathrm{Cu}\left(\approx 75^{\circ}\right) .^{22,23}$ The detection of polarization was performed using two different techniques. For the angle of $35^{\circ}$, the polarizer with a transmission of $30 \%$ was rotated manually between the measurements to record either the $\mathrm{P}$ or the $\mathrm{S}$ polarization of the reflected light. In this case, one assumes that the plasma remains stable within the measurements of $600 \mathrm{~s}$, but both spectra are recorded at the same position on the chip, using the optics A, so that the unpolarized transmission losses and spectral resolution are identical. Later, the manipulator was moved by about $20 \mathrm{~cm}$ away from the plasma source along the $\mathrm{z}$ axis to be placed in the line-of-sight of the angle of $70^{\circ}$. The polarization cube was used for splitting the signal into the $\mathrm{P}$ and $\mathrm{S}$ polarized components. The principle advantage of the second approach is the simultaneous measurements of both polarizations, being essential to prove the sensitivity of the Balmer lines to the polarization by light reflection. In the second case, however, the data for $\mathrm{S}$ and $\mathrm{P}$ polarization appear at different positions on the chip and thus have different transmission losses and slightly different spectral resolutions. Another disadvantage of the current measurements at $70^{\circ}$ is the angle of $20^{\circ}$ between the line-of-sight and the vacuum window, which results in different transmission for $\mathrm{S}$ and $\mathrm{P}$ polarized light emitted by the fast atoms. Nevertheless, both effects are of no relevance for the operation of the DSRM diagnostic as the reflectance of the surface is derived from the one spectrum only as the ratio of the red- to the blue-shifted signal. Figure 3 shows the details of the axial manipulator which carries the targets $\left(13 \times 13 \mathrm{~mm}^{2}\right)$. The water cooled target holder is completely covered by the ceramic plate (MACOR) including the pin with the length of $50 \mathrm{~mm}$, made of the stainless steel. Such a design of the manipulator was required to avoid the impact of emission of fast atoms generated from the target holder itself or the pin so that only the small target of interest remains at the negative potential. In contrast to the axial manipulator used in the previous measurements, the plasma terminates at the large target holder so that a new thermocouple also measuring the temperature during the plasma exposure was required.

Figure 4 shows the Balmer- $\alpha$ line emission using the $S$ polarizer at the angle of $70^{\circ}$, e.g., its axis of polarization is parallel to the target surface, and Fig. 5 shows the spectrum using the P polarizer. Both spectra are taken simultaneously. By inserting the axial manipulator

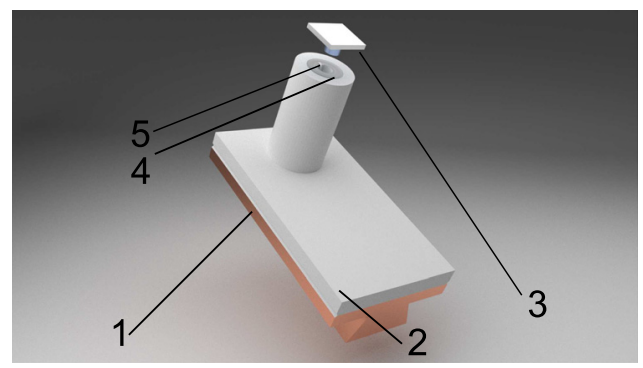

FIG. 3. The axial manipulator used for the detection of emission of fast atoms: 1-the target holder, 2-the ceramic plate, 3-the target of interest (Cu or Mo), 4-pin made of the stainless steel with the length of $5 \mathrm{~cm}$ inserted into the ceramic tube, and 5-thermocouple element.

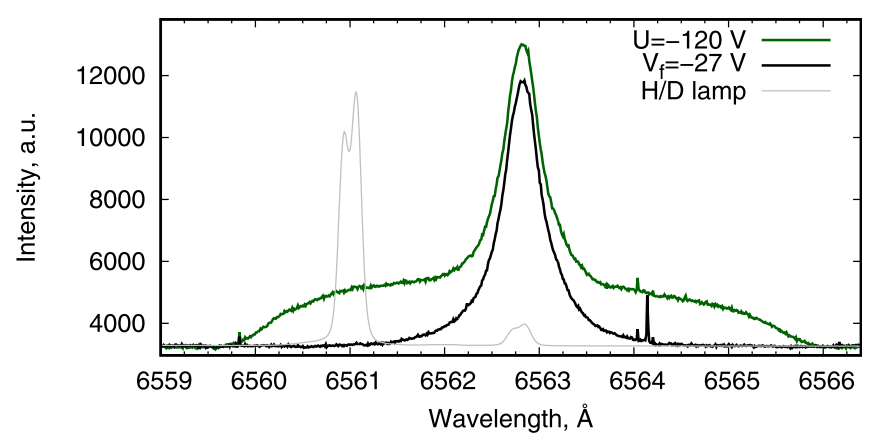

FIG. 4. Emission of the Balmer- $\alpha$ line in ArH plasma measured at the angle of $70^{\circ}$ in the front of Mo target using the $S$ polarizer. The measured spectrum at the applied potential of $-120 \mathrm{~V}$ is shown using the green line, and the spectrum measured at the floating potential of $-27 \mathrm{~V}$ is shown using the black line. The spectra of Balmer $\mathrm{D}_{\alpha}(6561.01 \AA)$ and $\mathrm{H}_{\alpha}(6562.282 \AA)$ lines using the calibration lamp containing $\mathrm{H}$ and $D$ gases installed in the front of the spectrometer are shown using the gray line.

into the plasma, the floating potential $V_{f}$ of $-27 \mathrm{~V}$ was measured on the target and the temperature of the target was $93^{\circ} \mathrm{C}$. The detected Balmer- $\alpha$ line is shown by the black curve. This low-energy (background) component is the most complex one in any kind of plasma. Its intensity ${ }^{24}$ and polarization ${ }^{25}$ are determined by plasma parameters, origin of emission, or excitation mechanisms in the plasma. In the case of PSI-2 plasma, this line is formed by dissociation of molecules, excitation by electrons, and the charge exchange between the ions and atoms. By applying the negative potential $U$ of $-120 \mathrm{~V}$, the reflected atoms become detectable: The kinetic energy of hydrogen atoms in the range of $100-200 \mathrm{eV}$ allows them to excite into $\mathrm{n}=3 \mathrm{lev}$ els by collision with Ar atoms in the ground or the metastable fraction. Finally, the Doppler effect separates the emission induced by the reflected fast atoms at the surface from the background line. The signal is shown using the green and orange lines in Figs. 4 and 5, respectively. As a result the emission induced by fast atoms contributes by about $20 \%$ to the intensity of the background component. Moreover, the integrals of the emission of fast atoms and the background component become even comparable.

Strictly speaking, the excitation of $\mathrm{H}$ atoms by collisions with $\mathrm{Ar}$ atoms in the ground state demonstrates the linear polarization on the order of $20 \%$ at the kinetic energy of $100 \mathrm{eV}$. However, in contrast to

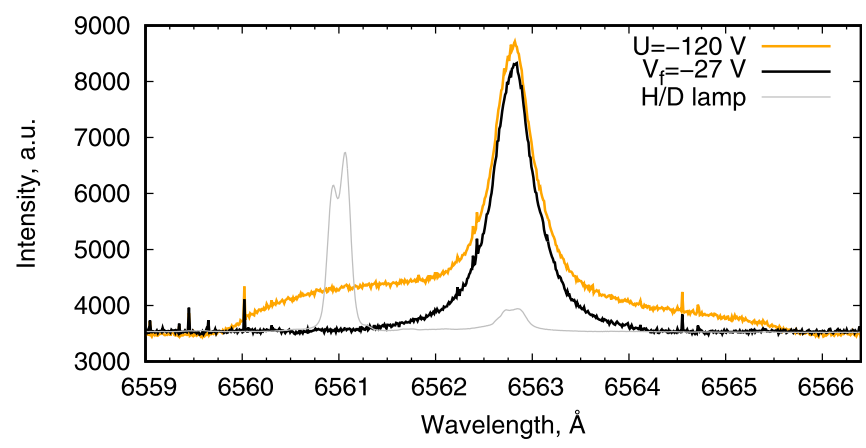

FIG. 5. Emission of the Balmer- $\alpha$ line in ArH plasma measured at the angle of $70^{\circ}$ in the front of the Mo target using the $\mathrm{P}$ polarizer (orange line). Other notations are the same as in Fig. 4. 
the beam-like experimental conditions, ${ }^{14}$ the reflected $\mathrm{H}$ atoms are back-scattered in the hemisphere of $2 \pi$ from the surface, and therefore, the polarization of observed emission must be further suppressed. One expects that the polarization of the red-shifted emission is solely determined by the polarization by light reflection of the surface as no signal induced by fast atoms approaching the target with pressures below $0.1 \mathrm{~Pa}$ exists. In order to exemplify this, the normalization on the blueshifted part of the emission from Figs. 4 and 5 was performed. The result confirms the expectation: Emission of Balmer- $\alpha$ in the front of the Mo target [Fig. 6(a)] demonstrates a drop of the red-shifted signal relative to the blue-shifted one by a factor of two. The observed variation of the red-shifted signal can be hardly explained in terms of excitation mechanisms in the plasma. According to Ref. 23, the P polarized reflectance of Mo is on the order of $20 \%$ only, compared to $80 \%$ for the $\mathrm{S}$ polarized signal. Thus, the polarization of light reflected at the metallic surface provides the most natural qualitative explanation of the experimental data. Nevertheless, in order to exclude any role of plasma or polarization properties of the vacuum windows and the front optics, the measurements were repeated for the $\mathrm{Cu}$ target as well. According to the optical properties of $\mathrm{Cu}$, not only the overall reflectance increases to $90 \%$ for the wavelength of the Balmer- $\alpha$ line, but also one expects a rather weak change in the polarization behavior. This is what exactly one observes in Fig. 6(b) again: (i) the red-shifted signal remains practically constant and (ii) both the spectra measured at the angle of $70^{\circ}$ in Fig. 6 (b) are practically symmetrical. Thus, the experimental spectra of emission of the Balmer- $\alpha$ line are at least in qualitative agreement with the polarization properties of targets of $\mathrm{Mo}, \mathrm{Cu}$, and $\mathrm{W}^{13}$

\section{MODELING OF EMISSION OF FAST ATOMS}

In order to obtain the degree of polarization for the arbitrary angle of observation $\theta_{0}$, a model needs to be introduced. We present
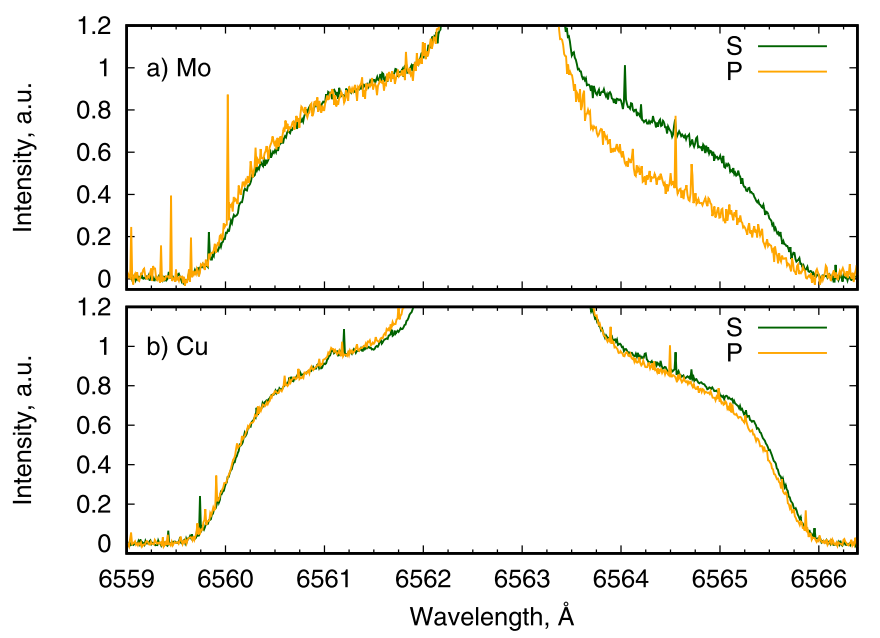

FIG. 6. Emission of the Balmer- $\alpha$ line in ArH plasma measured at the angle of $70^{\circ}$ in the front of Mo (a) and Cu (b) targets. The applied potential of $-120 \mathrm{~V}$ separates the background or cold component from the emission induced by fast atoms and reflectance. The $S$ polarized emission is shown using the green line, and the $P$ polarized emission is shown using the orange line. The normalization factor between the $\mathrm{S}$ and $\mathrm{P}$ polarized measurements was introduced for $\mathrm{Cu}(0.7)$ and Mo $(0.45)$ targets to depict the drop of the ratio between the red-and the blue-shifted signals. here the details of the model neglecting the temporal evolution of emission for the first $2-3 \mathrm{~mm}$ in the plasma, ${ }^{26}$ e.g., describing the emission in the steady-state phase only. Also, the fine-structure and the Zeeman splitting for the emission of fast atoms are neglected.

The blue-shifted emission of the reflected fast atoms can be described as

$$
\epsilon_{b}\left(\Delta \lambda, \theta_{0}\right)=\int_{v} f(\mathbf{v})\langle\sigma v\rangle d v^{3},
$$

where $\epsilon_{b}\left(\Delta \lambda, \theta_{0}\right)$ has the dimension of the excitation rate coefficient. It represents the convolution between the normalized distribution function of reflected atoms $f(\mathbf{v})$ with $\int_{l} f(\mathbf{v}) d v^{3}=1$ and the excitation rate coefficient of $\mathrm{H}$ atoms by collisions with $\mathrm{Ar}$ at the ground or metastable levels $\langle\sigma v\rangle$ detected at the Doppler shift $\Delta \lambda=\lambda-\lambda_{0}$ with $\Delta \lambda / \lambda_{0}=v \mathbf{e}_{\theta_{0}} / c$. Here $\mathbf{e}_{\theta_{0}}$ is the unit vector along the line-of-sight and $\sigma$ is the corresponding cross section. The spectral radiance and the line intensity of fast atoms can be further calculated as

$$
\begin{aligned}
L_{b}\left(\Delta \lambda, \theta_{0}\right) & =\frac{1}{4 \pi} \frac{h c}{\lambda} N_{H} N_{A r} l \epsilon_{b}\left(\Delta \lambda, \theta_{0}\right), \\
I_{b}\left(\theta_{0}\right) & =\int_{\Delta \lambda} L_{b}\left(\Delta \lambda, \theta_{0}\right) d(\Delta \lambda),
\end{aligned}
$$

where $l$ is the integration length along the line-of-sight, $h$ is the Planck constant, $N_{H}$ is the density of the reflected atoms, $N_{A r}$ is the density of Ar or its metastable fraction, $L_{b}\left(\Delta \lambda, \theta_{0}\right)$ is the spectral radiance, and $I_{b}$ is the line intensity. We note that the emitted power per unit volume and per unit sold angle $I_{b}\left(\theta_{0}\right) / l$ remains constant although the profile of emission varies as a function of angle. This fact was used to control the simulation.

The full modeling of the Doppler-shifted emission can be separated into an atomic and a kinetic part. The major advantage of experiments in low density plasma compared to the high density gas discharges is a relatively simple atomic physics description as practically only one rate coefficient is required, e.g., namely, the excitation of $\mathrm{H}$ by Ar atoms. The parametrization of the experimental data ${ }^{14}$ was done using the following expression for the Balmer- $\alpha$ and Balmer- $\beta$ lines:

$$
k=e^{-\left(p_{0} / E\right)^{p_{1}}} \cdot\left(p_{2}+p_{3} \ln (E)\right),
$$

and the results of the fit using formula (2) are shown in Fig. 7. We note that for the energies above $200 \mathrm{eV}$, another expression for the emission should be used. The thermal energy of Ar atoms is negligibly small compared to the kinetic energy of the reflected $\mathrm{H}$ atoms so that the rate coefficient is the product between the cross section and the velocity of $\mathrm{H}$ atoms. One expects that the temperature of Ar atoms remains close to the hydrogen background below $0.1 \mathrm{eV} .{ }^{10}$ The kinetic part of the distribution function of the reflected atoms is more complicated. First, we separated the distribution function in the angular and the energy dependent part

$$
f(\theta, E)=G(\theta) F(E) .
$$

For the angular distribution function, the expression from Ref. 15 was used,

$$
G(\theta)=\frac{1+b}{2 \pi} \cos ^{b}(\theta),
$$

where $\theta$ is the polar angle. The energy-dependent part was described using the following approximation: 


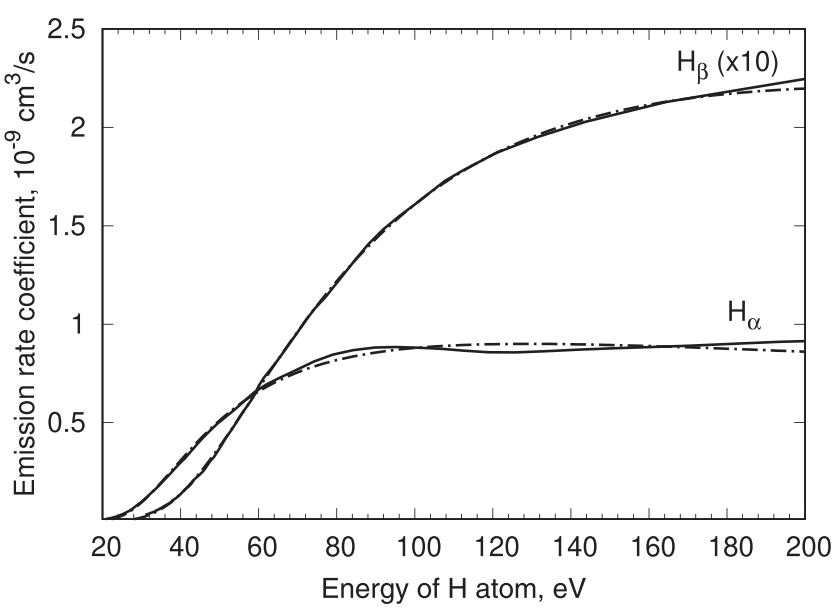

FIG. 7. Emission rate coefficients for the Balmer- $\alpha$ and the Balmer- $\beta$ (multiplied by a factor of ten) lines. The solid lines are experimental data from Ref. 14, and the dashed lines are the results of the fit. For the Balmer- $\alpha$ line, the coefficients $\left\{p_{i}\right\}=\left\{47.48,2.15,2.24 \times 10^{-9},-2.53 \times 10^{-10}\right\}$, and for the Balmer- $\beta$ line, the coefficients $\left\{p_{i}\right\}=\left\{83.3,1.67,7.29 \times 10^{-10},-8.54 \times 10^{-11}\right\}$.

$$
F(x)=A \frac{1-x}{\left(2-x_{0}-x\right)^{a}} .
$$

Here, $x=E / E_{0} \leq 1$ is a normalized dimensionless energy, $\mathrm{E}$ is the energy of reflected atoms, and $E_{0}=E_{0}\left(E_{i}, m_{1}, m_{2}\right)$ is the maximal energy of the reflected atom as a result of the binary collisions between the $\mathrm{H}$ atoms with mass $m_{1}$ and the target atom of $\mathrm{Mo}$ or $\mathrm{Cu}$ with the mass $m_{2},{ }^{17} E_{i}$ is the kinetic energy of ions impinging the surface. We note that in contrast to the Thompson distribution function of the sputtered particles for the reflected atoms, a unified description does not exist. The initial parameters of distribution function $A, x_{0}, a, b$ were obtained by parametrization of the output of the TRIM.SP for $\mathrm{Mo}$ and $\mathrm{Cu}$ at an incident energy of $\mathrm{H}$ ions of $100 \mathrm{eV}$. The position of the maximum of the energy distribution depends mostly on $x_{0}$ and its width on the $1 / a$ value. The parameter $A$ was used for the normalization of the integral $\int_{0}^{1} F(x) d x$. In the forthcoming analysis, neither the value $A$ nor the absolute values of the excitation rate coefficient plays the role as the DSRM diagnostics depends on the ratio of the red- to the blue-shifted signals only. The results of calculations using the SDTRIM.SP(4.15) code with $2 \times 10^{6}$ particles are exemplified in Figs. 8 and 9. The energy of the $\mathrm{H}^{+}$ions was assumed to be $100 \mathrm{eV}$ in these calculations, and the surface binding energy was $1 \mathrm{eV}$ for both elements. In Fig. 8, we show the angular distribution function for $\mathrm{H}$ on $\mathrm{Mo}$ and $\mathrm{H}$ on $\mathrm{Cu}$. The results show the overcosine distribution function with parameter $b \approx 1.5-1.6$ for both targets. The angular distribution could be well described by expression (4) except for those atoms emitted at the angles above $70^{\circ}$. However, the number of such atoms is relatively low compared to the angles of $45^{\circ}$. The calculated results reproduce the data of Ref. 27: in the energy range above $30 \mathrm{eV}$, and the overcosine distribution was expected for $\mathrm{H}$ impinging on $\mathrm{Ni}$ or $\mathrm{W}$ targets. By considering only the atoms in the energy range of $50-100 \mathrm{eV}$, the distribution function will be less peaked with $b \approx 1.36$. These data from the TRIM code validate our assumption on the separation of the distribution function into the energy and angular dependent parts, e.g., the parameter $b$ varies on the order of $20 \%-30 \%$. In the general

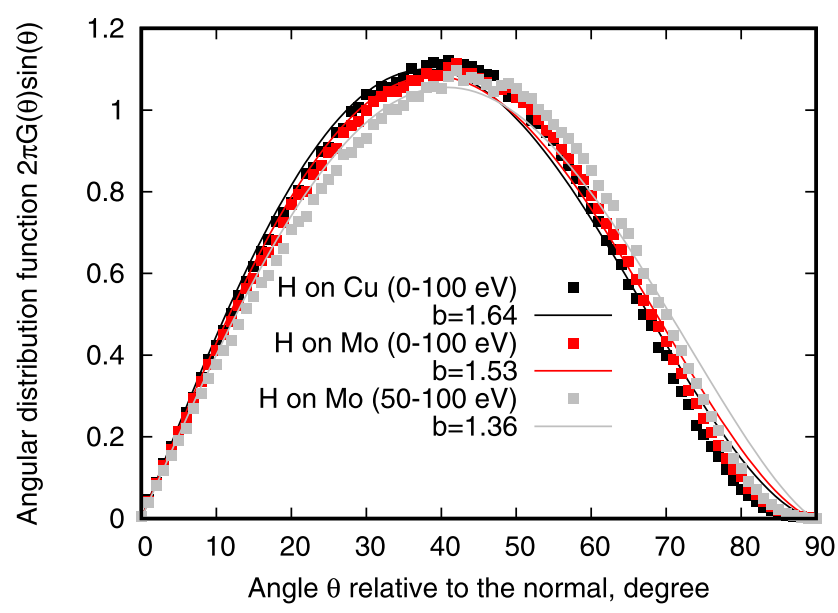

FIG. 8. Angular distribution function of reflected atoms $2 \pi \mathrm{G}(\theta) \sin (\theta)$ for $\mathrm{H}$ on $\mathrm{Mo}$ and $\mathrm{H}$ on $\mathrm{Cu}$ at the incident energy of $\mathrm{H}^{+}$ions of $100 \mathrm{eV}$.

case, such separation is not always possible as for instance shown for sputtered atoms. ${ }^{28}$ In Fig. 9, the energy distribution function of the reflected atoms is exemplified. Most of the atoms leave both targets with the average energy of $80-90 \mathrm{eV}$ and the width of the energy distribution corresponds to about $30-40 \mathrm{eV}$. In addition to the parameters of distribution function $\left(x_{0}, a, b\right)$ the scaling factors $C_{b, r}$ between the experimental and the modeled spectrum from Eq. (1), and the maximal energy of reflected atoms $E_{0}$ were considered as the free parameters in the fit. Whereas the ratio between $C_{r}$ and $C_{b}$ (index $r$ or $b$ refers to the red- or the blue-shifted part of the spectrum) defines the polarization properties of the mirror, the maximal energy of reflected atoms $E_{0}$ is of secondary importance. This value is known not better than with an accuracy of $10-20 \mathrm{eV}$ as the measurements of plasma potential $\phi_{p}$ using the Langmuir probe in mixed plasmas are rather uncertain

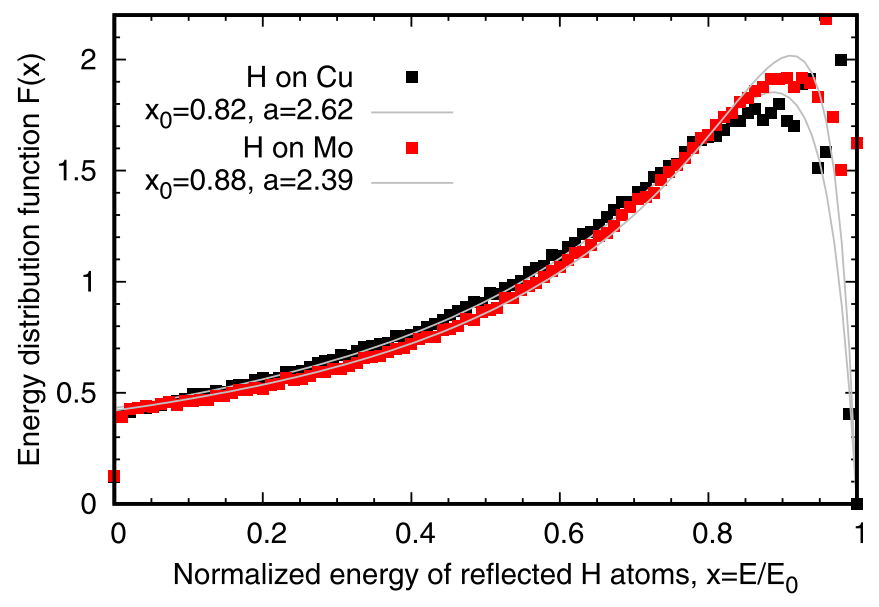

FIG. 9. Energy distribution function of reflected atoms $F(x)$ for $\mathrm{H}$ on $\mathrm{Mo}$ and $\mathrm{H}$ on $\mathrm{Cu}$ at the incident energy of the ions $E_{i}=100 \mathrm{eV}$. The calculated fraction of neutral particles $R_{N}\left(E_{i}\right)$ in the TRIM code equals 0.54 and 0.58 for $\mathrm{H}$ on $\mathrm{Cu}$ and $\mathrm{H}$ on $\mathrm{Mo}$, respectively. The data with points are the output from the TRIM code, and the gray lines are the result of the fit according to expression (5). 
$E_{i}=e\left(U-\phi_{p}\right)$. Also the uncertainty in the source of excitation of $\mathrm{H}$ atoms by collision with Ar atoms is still not resolved. We did not include the instrumental function, fine-structure separation, and the Zeeman effect in the modeling of fast atoms. On the one hand, the instrumental broadening $(3-5 \mathrm{eV})$ leads to the higher detected energy of reflected atoms. On the other hand, it was shown ${ }^{14}$ that the measured emission cross sections of the Balmer lines are mostly due to the population of $3 d$ levels of the reflected atoms. It will result in the asymmetry of the emission toward the red-shifted wavelengths compared to the statistical distribution. Finally, the presence of weak molecular lines deteriorates the accurate measurements of the onset of emission. ${ }^{29}$ Therefore, we left the detected maximal energy of reflected atoms as free parameters for the blue- and the red-shifted signals: $E_{0 b}$ and $E_{0 r}$. Thus the modeling of the blue-shifted component included four parameters $\left\{C_{b}, E_{0 b}, x_{0}, a, b\right\}$. For the red-shifted signal, only two free parameters were used $\left\{C_{r}, E_{0 r}\right\}$ as the distribution function must remain symmetrical in the measurements: The loss of the specular reflectance for the cooled target even after one hour of exposure was observed on the order of $5 \%$ only. ${ }^{12,13}$

The results of the modeling for the measurements of emission using the $\mathrm{S}$ direction of the linear polarizer at the applied potential of $-100 \mathrm{~V}$ are shown in Fig. 10. The model includes the simultaneous description of the emission induced by the reflected atoms and the background components according to Ref. 10. The initial profile of the simulations was obtained by the parametrization of the TRIM code data and convolution of the emission rate, distribution function, and Doppler effect.

A substantial disagreement between the measured (green profile) and the simulated emission profile of fast atoms using the TRIM data
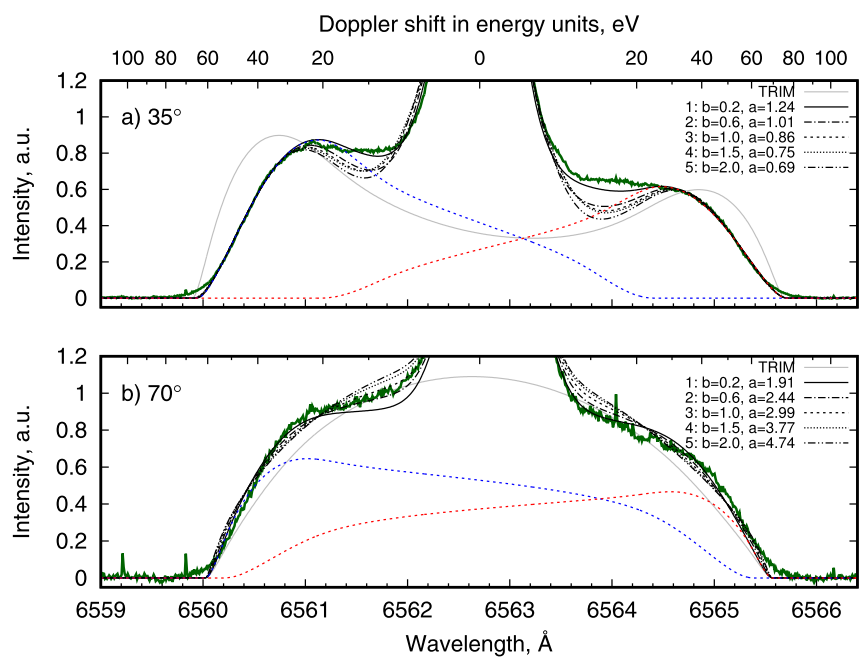

FIG. 10. Example of the modeling of the emission spectra measured at the angles of $35^{\circ}$ (a) and $70^{\circ}$ (b) using the $\mathrm{S}$ direction of the linear polarizer for the Mo target. The experimental spectra are shown in green, and the results of the modeling for different combinations of parameters of the angular and the energy distribution function $\left(x_{0}=0.85, a, b\right)$ are shown in black for different type lines. The emission profile assuming the distribution function from the TRIM code simulation is shown in gray $\left(x_{0}=0.85, a=2.5, b=1.5\right)$. The dot-dot blue and red curves exemplify the blue-shifted and red-shifted components of emission induced by fast atoms with $b=0.2$. (gray line) is detected by analyzing the emission at the angle of $35^{\circ}$. It seems that it is not possible to obtain the reasonable description of the measured emission although the intensities of the red and the blueshifted signals $C_{r}$ and $C_{b}$ were only guessed here. The numbers of reflected atoms with the energy of $50-100 \mathrm{eV}$ must be reduced, or the angular distribution of the reflected atoms has to be flattened in order to fit the experimental data. The parameters $C_{b, r}, a$, and $b$ were modified, and the value of $x_{0}=0.85$ was kept constant. Using the new set of parameters, the emission spectrum could be described reasonably well in the high energy range $(60-100 \mathrm{eV})$ for many combinations of parameters $b$ and $a$ irrespective of the question if the energy distribution determined by the parameter $a$ is physically correct or not in the low energy range. Thus, for instance, for $b \geq 1$, the parameter $a$ has to be reduced $(a<1)$ to describe the spectra; however, these solutions could be hardly acceptable. We note that the behavior of the function $F(x)$ at $x$ values below $40 \mathrm{eV}$ could not be traced due to the rapid drop of the excitation rate coefficients. By decreasing the parameter $b$ toward the values of $0.2-0.4$, even the low spectral energy range could be described reasonably well. Finally, for the values with $b=0.2$ and $a=1.24$, one obtains the most accurate description of the spectrum. In fact, the shown examples of the modeling demonstrate the major deficit of the simulation of Doppler shifted emission: Using one lightof-sight, only a number of solutions for angular and energy distribution are obtained, i.e., the results are ambiguous. The integrated characteristic of the emission does not allow a clear separation between the angular and the energy part. The description with $b=0.2$ shows that the number of energetic atoms is reduced compared to the TRIM results, but also the angular distribution is much broader as expected. The uncertainty in the distribution function of reflected atoms could be removed only by considering the emission at the angle of $70^{\circ}$ [Fig. 10(b)]. Also, for this angle, the model provides a rather good description of the emission. The difference between the experimental and the theoretical spectra exists only in the regions close to the unshifted component. The possible solutions with $b>1$ considered for the angle of $35^{\circ}$ must be rejected as the model overestimates the experimental spectra in the low energy intervals: The number of atoms which are reflected perpendicular to the target is higher as in the experiment. The solution with $b=0.6$ for the blue-shifted and with $b=0.2$ for the red-shifted interval demonstrates a rather good agreement with the experimental spectrum. The value of the parameter $a=1.91$ obtained at this line-of-sight is higher by about $50 \%$ compared to the angle of $35^{\circ}$. One of the possible reasons for this difference is a contribution of the light reflectance to the blue-shifted signal. The inaccuracy of the model in the description of the atoms moving parallel to the target, for instance, due to the separation into the energy and the angular part, results in the overestimation of the parameter $a$. In fact, the measurements with the $\mathrm{P}$ direction of polarization should provide identical values for the energy and angular distribution function as only the ratio between the normalization constant $C_{r} / C_{b}$ could be changed. One also expects a much better agreement for the parameter $a$ obtained from the both angles as the contribution of light reflectance to the blue-shifted part is suppressed. It is indeed observed in Fig. 11. Again, the values of parameter $b=0.2$ provide the most accurate description for both the lines-of-sight. The values of the parameter $a$ derived at the angle of $70^{\circ}$ approach those at $35^{\circ}$. In order to confirm that the value of parameter $b=0.2$ shows the most probable solution for both the angles, we varied the parameter $x_{0}$ as well and investigated 

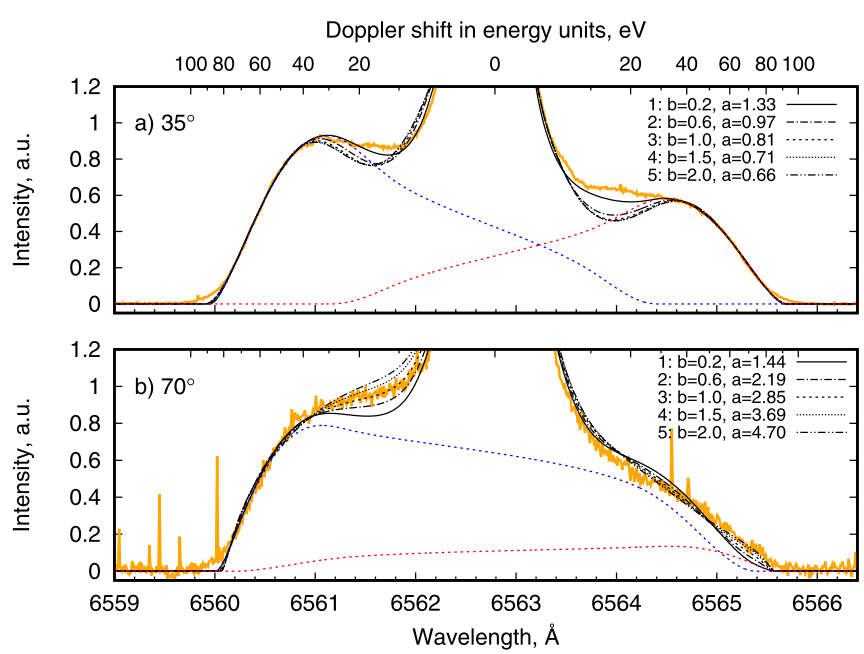

FIG. 11. Example of the modeling of the emission spectra measured at the angle of $35^{\circ}$ (a) and $70^{\circ}$ (b) using the $\mathrm{P}$ direction of the linear polarizer for the Mo target. The experimental spectra are shown in orange, and the results of the modeling for different combinations of parameters of the angular and the energy distribution function $\left(x_{0}=0.85\right)$ are shown using black lines. The dot-dot blue and red curves exemplify the blue-shifted and red-shifted components of emission induced by fast atoms with $b=0.2$.

the behavior of different combinations $\{a, b\}$ for both lines-of-sight for the data using the $\mathrm{P}$ polarizer. The results of calculations at different values of the parameter $x_{0}$ are summarized in Fig. 12. The data for the observation angle of $70^{\circ}$ are shown using the solid lines and for $35^{\circ}$ using the dashed lines. The general behavior of the curves confirms the results in Figs. 10 and 11. So, for instance, for the angle of $35^{\circ}$, one could compensate the high $b$ values by lowering the number of particles with high energy and reducing the parameter $a$. For the angle of $70^{\circ}$, both the parameters must be increased simultaneously to describe the spectra of fast atoms. The curves approach or cross each other for the range of parameters $a=1.2-1.7$ and $b=0.1-0.2$.

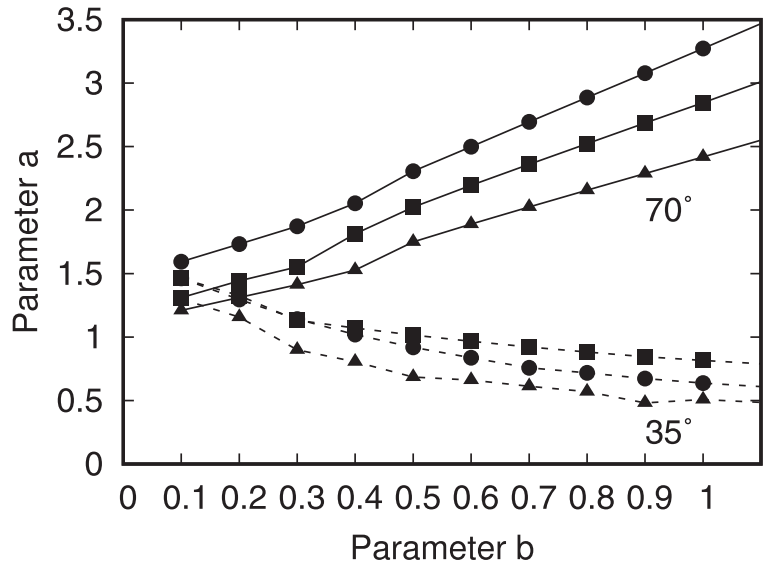

FIG. 12. Parameters of the energy and angular distribution functions obtained by fitting the spectra at the angles of $35^{\circ}$ and $70^{\circ}$ for different values of $x_{0}: x_{0}=0.8$ (lines with circles), $x_{0}=0.85$ (lines with squares), and $x_{0}=0.9$ (lines with triangles).
The ratio of coefficients $C_{r} / C_{b}$ defines the polarization of the Mo target using the emission at the Balmer line. Figure 13 summarizes the obtained values of polarization and compares them with the theoretical values. First of all, the experimental data demonstrate a relatively weak dependence on the parameter of the angular distribution $b$. For instance, the solution with different $b$ values varying from 0.2 to 1.0 results in the variation of reflectance not more than $7 \%$ for both polarizations at the angle of $35^{\circ}$. In the case of very weak reflectance measured at the angle of $70^{\circ}$, the variation is much stronger and one obtains the values ranging from 0.17 to 0.3 . In fact, it shows that for materials with high reflectance, the measured values could be derived even with unknown parameters of distribution functions. It could occur if for instance only one line-of-sight is available. In all cases, the increase in the $b$ value stimulates stronger separation between the nonpolarized (blue-shifted) and polarized (red-shifted) signals so that the reflectance increases in all cases. The agreement between the measured and the theoretical data for both angles is found within 15\% (except for $\mathrm{P}$ polarization at the angle of $70^{\circ}$ ).

\section{DISCUSSION AND CONCLUSION}

In this paper, we investigated the impact of polarization by reflection on the line shapes of Balmer lines induced by reflected atoms in low density $\mathrm{ArH}$ gas discharges. It was shown that if one observes the Balmer lines close to the Brewster angle in the front of metallic (conductive) surfaces, the polarization causes a significant impact on the line shapes. The effect depends on the materials of the mirror, for instance, the strong variation of the red-shifted part in the spectra of $\mathrm{Mo}$ and $\mathrm{W}^{13}$ on the order of $50 \%$ is detected, whereas for $\mathrm{Cu}$, a very weak variation is measured. The results correlate clearly with the optical properties of the materials. In contrast to our previous measurements at $35^{\circ}$, for large angles of observations with respect to the surface normal, the values of polarization can be derived only using the appropriate models: The overlap between the direct and reflected
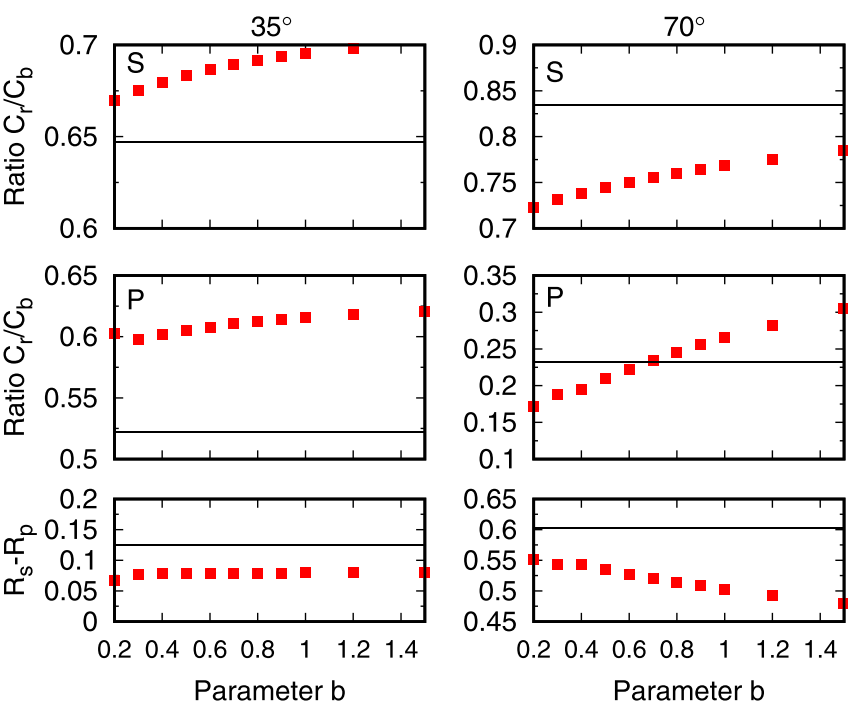

FIG. 13. Polarization of reflectance defined as the ratio between the red- $\left(C_{r}\right)$ and the blue-shifted $\left(C_{b}\right)$ emission (red points) and the theoretical values from Ref. 23 (black line). The top panel corresponds to the $S$ polarization $R_{S}$, the middle panel to the $\mathrm{P}$ polarization $R_{p}$, and the lowest panel to the difference $R_{s}-R_{p}$. 
light is too strong to use the ratio between the corresponding integrals. ${ }^{12}$

Using one angle of observation, only the number of solutions for energy and angular distribution could sufficiently well describe the spectrum. However, by using another angle of observation, many combinations must be dropped. The angular distribution with parameter $b \approx 0.2$ provides the best description of the spectra measured for the Mo surface for both angles and for both polarizations. The number of high energy reflected atoms is less than that the TRIM code provides, e.g., the parameter $a$ has values between 1.2 and 1.9. We note that only the emission of the atoms with the energy above $40-60 \mathrm{eV}$ could be traced using this technique. The disagreement with the TRIM code input is obvious; however, one should take into account that the simulations do not consider either the ion implantation into the material or the surface morphology or retention of hydrogen. We are not aware of any other results in the energy range of $100 \mathrm{eV}$ of incident $\mathrm{H}$ ions to be compared with. At the energy of $2000 \mathrm{eV}$, the angular distribution of $\mathrm{H}$ on AuPd was found on the order of $b \approx 0.6$ in Ref. 15, confirming broader angular distribution compared to the diffusive one.

The degree of polarization by reflection at the Balmer- $\alpha$ line is in good agreement with the theoretical data (15\%) using $b=0.2$ (Fig. 13). Since the results are very weak functions of the exact distribution function of the reflected atoms, the DSRM diagnostic is especially attractive for future applications. The derived polarization increases by peaking the angular distribution of reflected atoms. So, for instance, for the observation angle of $70^{\circ}$, the value of $S$ polarization increases from 0.72 to 0.78 only. However, the difference is large for rather weak values of polarization as it is difficult to fit the red-shifted emission accurately. The values of polarization are found systematically higher for the angle of $35^{\circ}$ and systematically lower for $70^{\circ}$. In the first case, for instance, the measurements provide the values of polarization of 0.67 compared to $0.65(\mathrm{~S})$ and 0.63 compared to $0.53(\mathrm{P})$. For the angle of $70^{\circ}$, the situation is the opposite; the derived values of polarization are underestimated with 0.72 compared to 0.83 (S) and 0.17 compared to $0.23(\mathrm{P}) .^{23}$ Such a systematic offset is most probably connected with the limitation of the experimental setup but not with the approach itself. Indeed, as exemplified in Fig. 1, the measurements at different angles are also made from the different sides of the plasma PSI-2, including the axial shift of the target. It could be partially confirmed by analyzing the difference in the $R_{s}-R_{p}$ values. In this case, the offset in the measurements must be partially removed. The difference between the measured and the theoretical data is on the order of 0.05 . We should mention that the most recent theoretical data ${ }^{30}$ show the values of $0.28(n=3.17, k=3.7)$ for the P reflectance at $650 \mathrm{~nm}$, reducing the theoretical results $R_{s}-R_{p}$ closer to our values.

The measurements of polarization by light reflection at metallic surfaces represent the powerful enhancement to the DSRM diagnostic. Without doubt, it is hardly possible to obtain the polarization degree with the same precision as with the help of specialized instruments. However, such measurements could be applied if the status of a mirror or a plasma facing surface can be monitored using the one line-ofsight only. In this case, the emission induced by reflected fast atoms represents one of the few if not the only available technique to perform this task in the absence of other light sources.

\section{ACKNOWLEDGMENTS}

We thank S. Brezinsek for careful reading of this manuscript and valuable comments and suggestions. This work has been carried out within the framework of the EUROfusion Consortium and received funding from the Euratom research and training program 2014-2018 and 2019-2020 under Grant Agreement No. 633053. The views and opinions expressed herein do not necessarily reflect those of the European Commission.

\section{REFERENCES}

'E. Garcia-Caurel, A. De Martino, J.-P. Gaston, and L. Yan, Appl. Spectrosc. 67, 1 (2013).

${ }^{2}$ A. Marvin, F. Toigo, and V. Celli, Phys. Rev. B 11(8), 2777 (1975).

${ }^{3}$ T. Fujimoto, Plasma Phys. Controlled Fusion 41, A625 (1999).

${ }^{4} \mathrm{Ph}$. Mertens, S. Brezinsek, P. T. Greenland, J. D. Hey, A. Pospieszczyk, D. Reiter, U. Samm, B. Schweer, G. Sergienko, and E. Vietzke, Plasma Phys. Control. Fusion 43(12A), A349 (2001).

${ }^{5}$ F. M. Levinton and H. Yuh, Rev. Sci. Instrum. 79, 10F522 (2008).

${ }^{6}$ E. Stambulchik and Y. Maron, Phys. Rev. Lett. 113, 083002 (2014).

${ }^{7}$ O. Marchuk, Yu. Ralchenko, R. K. Janev, W. Biel, E. Delabie, and A. M. Urnov, J. Phys. B: At. Mol. Opt. Phys. 43, 011002 (2010).

${ }^{8} \mathrm{Ph}$. Mertens, J. Fusion Energy 38, 264 (2019).

${ }^{9}$ J. Howard, C. Michael, H. Chen, R. Lester, A. Thorman, and J. Chung, J. Instrum. 10, P09023 (2015).

${ }^{10}$ O. Marchuk, C. Brandt, A. Pospieszczyk, M. Reinhart, S. Brezinsek, B. Unterberg, and S. Dickheuer, J. Phys. B.: At. Mol. Opt. Phys. 51, 025702 (2018).

${ }^{11}$ O. Marchuk, C. Brandt, and A. Pospieszczyk, "Verfahren zur Bestimmung der Oberflächeneigenschaften von Targets," German patent DE102016002270B3 (2017).

${ }^{12}$ S. Dickheuer, O. Marchuk, C. Brandt, A. Pospieszczyk, A. Goriaev, M. Ialovega,

B. Göths, Y. Krasikov, A. Krimmer, P. Mertens, and A. Kreter, Rev. Sci. Instrum. 89, 063112 (2018).

${ }^{13}$ S. Dickheuer, O. Marchuk, S. Ertmer, A. Goriaev, M. Ialovega, B. Göths, Y. Krasikov, P. Mertens, and A. Kreter, Nucl. Mater. Energy 17, 302 (2018).

${ }^{14}$ B. Van Zyl, M. W. Gealy, and H. Neumann, Phys. Rev. A 31, 2922 (1985).

${ }^{15}$ A. V. Phelps, Phys. Rev. E 79, 066401 (2009).

${ }^{16}$ N. Cvetanović, B. M. Obradović, and M. M. Kuraica, J. Appl. Phys. 105, 043306 (2009).

${ }^{17} \mathrm{H}$. Verbeek, "Backscattering of light ions from metal surfaces," in Radiation Effects on Solid Surfaces, edited by M. Kaminsky (American Chemical Society, 1976), Vol. 158, p. 245.

${ }^{18}$ P. C. Stangeby, The Plasma Boundary of Magnetic Fusion Devices (IoP Publishing, Ltd., Bristol and Philadelphia, 2000), p. 79.

${ }^{19}$ J. F. Ziegler, "SRIM/TRIM code," see www.srim.org.

20 J. P. Biersack and W. Eckstein, Appl. Phys. A 34, 73 (1984).

${ }^{21}$ A. Kreter, C. Brandt, A. Huber, S. Kraus, S. Möller, M. Reinhart, B. Schweer, G. Sergienko, and B. Unterberg, Fusion Sci. Technol. 68, 8 (2015).

${ }^{22}$ M. N. Polyanskiy, "Refractive index database," see https://refractiveindex.info (last accessed December 14, 2018).

${ }^{23}$ W. S. M. Werner, K. Glantschnig, and C. Ambrosch-Draxl, J. Phys. Chem. Ref. Data 38, 1013 (2009).

${ }^{24}$ N. M. Šišović, G. Lj. Majstorović, and N. Konjević, Eur. Phys. J. D 41, 143 (2007).

${ }^{25}$ G. Sergienko, G. I. Stotsky, and N. M. Zykova, Meas. Sci. Technol. 5, 1448 (1994).

${ }^{26}$ C. Brandt, O. Marchuk, A. Pospieszczyk, and S. Dickheuer, AIP Conf. Proc. 1811, 130001 (2017).

${ }^{27}$ W. Eckstein and J. P. Biersack, Appl. Phys. A 38, 123-129 (1985).

${ }^{28}$ M. Stepanova and S. K. Dew, J. Vac. Sci. Technol., A 19, 2805 (2001).

${ }^{29}$ B. P. Lavrov and I. S. Umrikhin, J. Quant. Spectrosc. Radiat. Transfer 182, 180 (2016).

${ }^{30}$ M. Minissale, G. T. Zeweldi, R. Bisson, and L. Gallais, Nucl. Fusion 58, 096012 (2018). 